\title{
Real-Time Tissue Elastography in Epidermal Cyst: along with Enhanced MRI and Elasticity Features of Peripheral Rim
}

\author{
Kazumi Fujioka* \\ Department of Dermatology, Fujioka Dermatological Clinic, Japan
}

*Corresponding author: Kazumi Fujioka, Department of Dermatology, Fujioka Dermatological Clinic, 1-35-10, Tamagawa, Ota-ku, Tokyo, Japan

ARTICLE INFO

Received: 蔧 March 30, 2019

Published: April 04, 2019

Citation: Kazumi Fujioka. Real-Time Tissue Elastography in Epidermal Cyst: along with Enhanced MRI and Elasticity Features of Peripheral Rim. Biomed J Sci \& Tech Res 16(5)-2019. BJSTR. MS.ID.002904.

Keywords: Real-Time Tissue Elastography; Epidermal Cyst; Peripheral Rim; High-Resolution Ultrasonography; Fibrosis

\begin{abstract}
The development of more potent machines and high-frequency probes has contributed to a daily practice and research study in the dermatologic field. The minimum frequency recommended by the international working group, called DERMUS (Dermatologic Ultrasound) for performing dermatologic examinations was $15 \mathrm{MHz}$. The author previously described that the comprehensive procedure using high-resolution ultrasound is useful for the accurate diagnosis of epidermal cyst. In this article, epidermal cyst features on Real-time Tissue Elastography (RTE) along with enhanced MRI and elasticity features of peripheral rim is reviewed in detail. It is suggested that epidermal cyst is relatively soft nature, but it is plausible that epidermal cyst is harder nature than lipoma on RTE. Thin and smooth rim on enhanced MRI with soft nature on RTE, having high elasticity, indicate no fibrosis, reflecting the possibility of the unruptured condition. The author will suggest that thin and smooth on enhanced MRI and soft nature on RTE in peripheral rim features contribute accurate diagnosis in unruptured epidermal cyst.
\end{abstract}

Abbreviations: RTE: Real-time Tissue Elastography; US: Ultrasonography; HPV: Human Papillomavirus; SWE: Shear-Wave Elastography

\section{Introduction}

In recently years, dermatologic ultrasound imaging has been rapidly developed [1]. DERMUS (Dermatologic Ultrasound), the international working group, was formed and provided the guidelines for performing dermatologic ultrasound examinations [1] and proposed for an assessment training program [2]. Epidermal cyst are common benign subcutaneous lesions known to result from progressive cystic ectasia of the infundibular portion of hair follicles [3]. Epidermal cyst also develops in hairless skin such as planter and palmer. A different pathogenesis was suggested in the occurrence in hair follicles and in hairless skin namely palmoplantar location. It is sometimes difficult to distinguish an epidermal cyst from other benign skin tumors, such as lipoma, ganglia, pilomatricoma and dermatofibroma. Meanwhile, asymptomatic unruptured epidermal cysts need no treatment, whereas ruptured or infected cysts should be surgically removed [4].Therefore preoperative correct diagnosis is important, and different treatment options should be planned for ruptured and unruptured cysts [5].

Real-time sonoelastography is an ultrasound-based technique using the property that the tissue strain due to compression is lower in hard tissues [6]. Real-Time Tissue Elastography (RTE) is widely used for diagnosis of breast [7], thyroid [8] and testis [9] on tissue elasticity. With respect to the differentiation the ruptured from unruptured, characteristic features on gray-scale Ultrasonography (US) $[10,11]$ and color Doppler US $[11,12]$ have been reported. The author previously reported that the comprehensive procedure using high-resolution ultrasound is useful for the accurate diagnosis of epidermal cyst [13]. The studies have been reported on RTE of epidermal cyst, using RTE [5,9,13-15] and Shear-Wave Elastography (SWE) [16]. In this article, the current knowledge of epidermal cyst on RTE along with enhanced MRI and elasticity features on RTE 
of peripheral rim will be reviewed in detail. The author also will describe the differentiating ruptured from unruptured epidermal cyst with an emphasis of the peripheral rim.

\section{Dermatologic Ultrasound}

Dermatologic ultrasound imaging has been rapidly growing in recently years because of the development of high-resolution multifrequency transducers and multichannel color Doppler machines [1]. DERMUS proposed that the minimum frequency recommended for performing dermatologic examinations was 15 $\mathrm{MHz}$ [1]. We usually perform US tests for dermatologic diseases with a high-resolution, broad-band $(5 \mathrm{MHz}-18 \mathrm{MHz})$ linear transducer (Nobulus Hitachi, Ltd.Tokyo, Japan). Until now we have reported many studies in the dermatologic field [13,17-22].

\section{Pathogenesis in Epidermal Cyst}

Epidermal cyst are common benign subcutaneous lesions known to result from progressive cystic ectasia of the infundibular portion of hair follicles [3]. While in hairless skin such as planter and palmer regions, it has been proposed that palmoplantar epidermoid cyst may be related eccrine ducts and that human papillomavirus (HPV) 60 may play a role in their pathogenesis [23]. It has been also reported that palmoplantar epidermal cyst may develop when infected by HPV 57 or 60 [24]. A different pathogenesis was suggested in the occurrence in hair follicles and in hairless skin. The imaging appearance of HPV-associated plantar epidermoid cysts on MR and US has been reported [25].

\section{Gray Scale Ultrasonographic Appearances}

It has been reported that epidermal cyst on gray-scale US shows 2 types as pseudotesis and nonpseudotestis [26] and the report of the classification of 5 patterns have been described [27]. Unruptured cyst shows round-to-oval shape, posterior echo enhancement, and lateral shadowing [5]. In relation to the halo features, it has been reported that the presence of halo on grayscale US is detected in $67 \%$ in all epidermal cyst [10], while the presence of a halo represents $61.5 \%$ in unruptured type [4]. They suggested that a higher frequency of the absence of a halo was statistically recognized in the ruptured cysts than in the unruptured ones [4]. The hypoechoic halo of epidermal cyst might be consistent with the more complete walls of cysts composed of keratinizing stratified squamous epithelium. Whereas ruptured cyst represents lobulated shape, a poorly defined boundary, absence of a halo [4]. Pericystic change has been also detected in ruptured type [11]. Figure 1a shows pseudotestis pattern with filiform anechoic areas, echogenic reflectors and hypoechoic rim on gray-scale US, which is pathologically confirmed in the right mandibular location.

\section{Color Doppler Utrasonograpic Appearances}

Unruptured type shows usually no substantial blood flow, while pericystic changes are demonstrated in ruptured type on grayscale US and color Doppler US shows pericystic changes and/or peripheral vascularity $[11,12]$. A few peripheral blood flow signals were depicted on color Doppler US (Figure 1b).
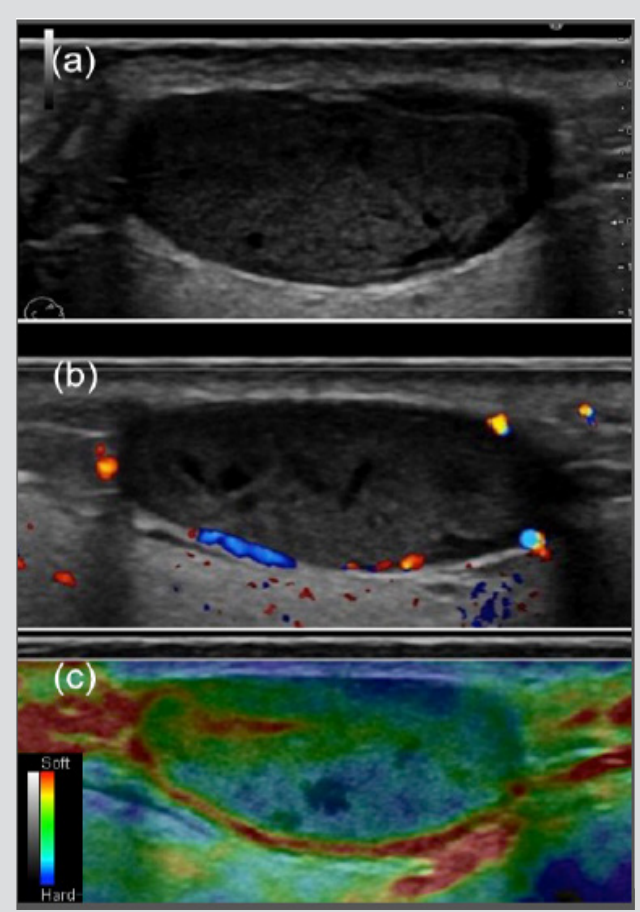

Figure 1: Pseudotestis type of epidermal cyst on gray scale US pathologically diagnosed in the right mandibular location in a 41-year-old man.

a) On gray-scale US shows pseudotestis pattern with filiform anechoic areas, echogenic reflectors and hypoechoic rim.

b) A few peripheral blood flow signals on rim are depicted on color Doppler US.

c) Epidermal cyst shows more green than blue, peripheral rim represents green to red color consistent with soft nature, suggesting without fibrosis condition on Real-Time Tissue Elastography. 


\section{Real-time Tissue Elastography Appearances}

Real-time sonoelastography is an ultrasound-based technique using the property that the tissue strain due to compression is lower in hardness tissues [6]. RTE is widely used for diagnosis of breast [7], thyroid [8] and testis lesions [9] on tissue elasticity. Typically, red is used to represent softer tissues, blue represents harder tissues, and yellow or green shows intermediate elasticity [28]. While shearwave elastography (SWE) namely termed dynamic elastography, is based on the measurement of the propagation velocity distribution of the directional shear wave, produced by an ultrasound pulse [28]. It is sometimes difficult to distinguish an epidermal cyst from other benign skin tumors, such as lipoma, ganglia, pilomatricoma and dermatofibroma. Some studies have been reported on RTE [5,13-15] and on SWE [16]. Lee et al. concluded that the stain ratios of lipoma, ganglia, were statistically different from epidermal inclusion cysts but there was no statistically difference between epidermal inclusion cyst and pilomatoricomas [14]. Park et al. described that the strain elastography score was classified into four types.

Their data recognized variable elasticity of epidermal cyst. They indicated that superficial epidermoid tumor shows a softer nature than dose malignant tumor; however, it does not have a different SE pattern from other benign tumors [15]. The author previously reported the nonpseudotestis type of epidermal cyst which was pathologically confirmed in the plantar location [13]. Image features on RTE showed more green than blue, suggesting relatively soft nature [13]. Nodule demonstrated more green than blue, showing relatively soft nature, while the peripheral rim showed green to red color consistent with soft nature, suggesting no fibrotic condition (Figure 1c). Our data indicates that epidermal cyst is relatively soft nature, but it seems to be harder than lipoma. With respect to the ruptured study, it has been studied that $\mathrm{R}$ value inside the cyst (Rinside), $R$ value outside the cyst (Routside) and Rinside/Routside ratio, which shows the ratio of the elasticity inside and outside the cyst, were examined on sonoelastography [5]. They concluded that RTE is able to detect differences in tissue elasticity between ruptured and unruptured epidermal cysts [5]. On SEW, it is reported that epidermal cyst statistically showed a greater median shear modulus than ganglion cysts and lipomatous tumors [16]. Meanwhile, it has been reported that neurogenic tumors or neuromas, dermoid cysts, and metastatic tumors were considered as high stiffness, namely low elasticity, while lipoma, lymphatic and/or venous maiformations, and cystic tumors show low stiffness, so-called high elasticity [28,29].

\section{Peripheral Rim Appearances}

The rim on gray-sale US showed a sharply defined hypoechoic or hyperechoic rim surrounding the epidermal cyst. Kim et al reported that peripheral low echoic rim, consistent with the capsule was detected in $67 \%$ on gray-scale US [10]. Yuan et al. have statistically emphasized a higher frequency of the absence of a halo in the ruptured cysts than in unruptured cysts [4]. The hypoechoic halo of ruptured and unruptured epidermal cysts might represent the more complete walls of cysts composed of keratinizing stratified squamous epithelium $[4,11,26]$. It has been suggested that if the fragmented wall of a ruptured epidermal cyst is involved with an inflammatory reaction or granulation tissue, the sharpness and continuity of the walls would pathologically disappear, and the halos on gray-scale US would be absent [4]. With respect to peripheral rim features on RTE, there have not been reported bibliographically. Soft nature of rim appearance, showing without fibrosis may be consistent with unruptured type. Rim feature on RTE may contribute to determine the accurate diagnosis in differentiation unruptured type from ruptured type.

TRE procedure is evaluated using elasticity state not only in malignancy grade but also in fibrosis condition. Thin and smooth rim with soft nature, having high elasticity on RTE indicate no fibrosis, reflecting the possibility of the unruptured condition. In relation to MRI findings, it is reported that epidermal cyst shows high signal intensity on $\mathrm{T} 2$ and all peripheral rim enhancement [10]. Thin and smooth rim enhancement was shown in unruptured type, while the thick and irregular rim enhancement was demonstrated in ruptured type [30]. They also have noted that most ruptured epidermal cyst contained septa and showed thick and irregular rim enhancement and fuzzy adjacent soft-tissue enhancement [30]. Fisher et al. have suggested that the rim enhancement might be due to fibrosis and foreign body reaction around the cyst wall remnants and keratinous material [31]. Axial T2-weighted image shows hyperintense mass which was pathologically diagnosed as epidermal cyst in the right mandibular location (Figure 2a). Thin and smooth rim enhancement was also shown on contrastenhanced T1-weighted image, suggesting unruptured type of epidermal cyst (Figure $2 \mathrm{~b}$ ).

\section{Pathological Appearances}

If epidermal cyst rupture, suppuration, foreign body granulomatous reaction, and granulation tissue can be recognized [32]. It has been reported that the contents of the cysts in the ruptured cyst included more abscess, parakeratotic cells, red blood cells, giant cells and pilomatoricoma-like cells than in the unruptured type [33]. With respect to cystic wall, hyperplasia, hypergranulosis, microabscess, parakeratosis, necrotic change, verrucous changes and exocytosis, which were revealed more in the ruptured type than in the unruptured one [33] Figure 2c showed cystic lesion lined by stratified squamous epithelium with variable amounts of keratinous debris and packed keratin lamellae. Cystic lesion with a stratified squamous epithelial lining, keratinous debris, multinucleated giant cells, and inflammatory reactions were also observed (Figure 2d). 


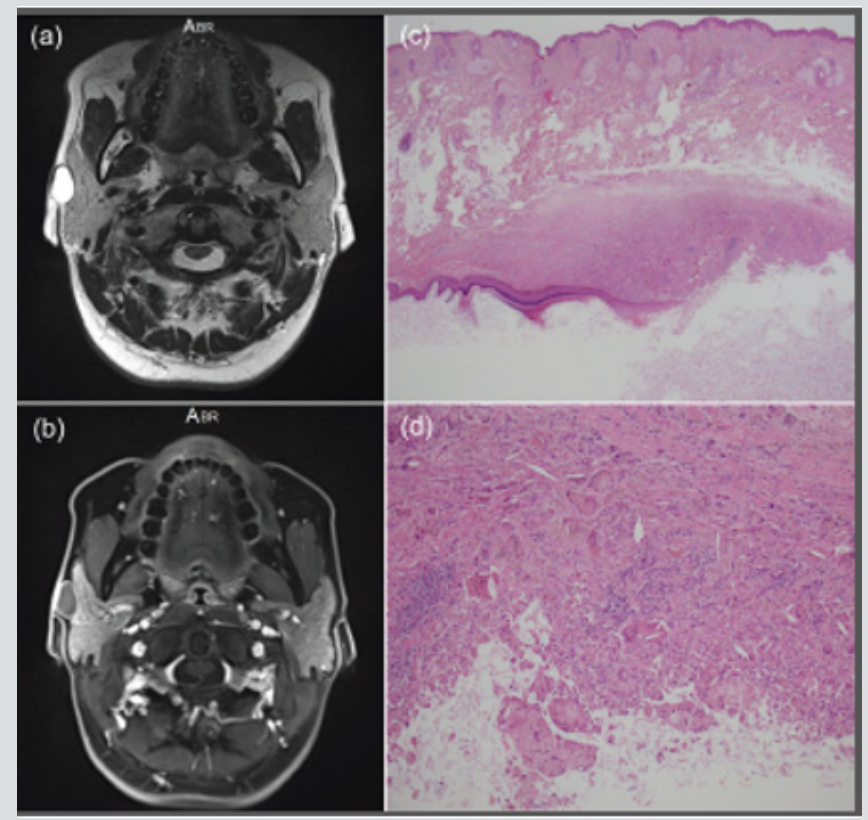

Figure 2: Pseudotestis type of epidermal cyst on gray-scale US pathologically diagnosed in the right mandibular location in a 41-year-old man.

a) Axial T2-weighted image shows hyperintense mass.

b) Thin and smooth rim enhancement is shown on contrast-enhanced T1-weighted image.

c) Cystic lesion lined by stratified squamous epithelium with variable amounts of keratinous debris and packed keratin lamellae are noted (hematoxylin-eosin, original magnification x10).

d) Cystic lesion with a stratified squamous epithelial lining, keratinous debris, multinucleated giant cells, and inflammatory reactions are shown (hematoxylin-eosin, original magnification $\times 50$ ).

It is suggested that epidermal cyst is relatively soft nature, but it is plausible that epidermal cyst is harder nature than lipoma on RTE. In relation to the ruptured state, it may be helpful finding that ruptured type is softer nature than unrptured type. Peripheral rim on gray-scale US statistically seems to be detectable in unruptured type than in ruptured epidermal cyst. Thin and smooth rim on enhanced MRI and soft nature on RTE, having high elasticity, indicate no fibrosis, reflecting the possibility of the unruptured condition. In addition to the enhanced MRI, evaluation of the elasticity on RTE in peripheral rim is useful for correct diagnosis in differentiating ruptured from unruptured type.

\section{Conclusion}

a) The author suggests epidermal cyst is relatively soft nature, but it is plausible that epidermal cyst is harder nature than lipoma on Real-Time Tissue Elastography.

b) Thin and smooth rim on enhanced MRI with soft nature on Real-Time tissue Elastograhy, having high elasticity, indicate without fibrosis, reflecting the possibility of the unruptured condition.

c) The author will suggest that thin and smooth on enhanced MRI and soft nature on Real-Time Tissue Elastography in peripheral rim features contribute accurate diagnosis of unruptured epidermal cyst.

\section{Acknowledgement}

The author appreciates Dr. Akira Fujioka, Dr. Minoru Oishi, and Dr. Ken Hayashi.

\section{References}

1. Wortsman X, Alfageme F, Roustan G, Arias-Santiago S, Martorell A, et al. (2016) Guidelines for performing dermatologic ultrasound examinations by the DERMUS group. J Ultrasound Med 35(3): 577-580.

2. Wortsman X, Alfageme F, Roustan G, Arias-Santiago S, Martorell A, et al. (2016) Proposal for an assessment training program in dermatologic ultrasound by the DERMUS group. J Ultrasound Med 35(11): 2305-2309.

3. Kirkham N (2005) Tumors and cysts of the epidermis. In: Elder DE, Elenitsas R, Johnson Jr BL, Murphy GF (Eds.). Lever's histopathology of the skin. $9^{\text {th }}$ (edn.). Philadelphia: Lippincott Williams \& Wikins, pp. 814815.

4. Yuan WH, Hsu HC, Lai YC, Chou YH, Li AF (2012) Differences in sonographic features of ruptured and unruptured epidermal cysts. J Ultrasound Med 31(2): 265-272.

5. Park J, Chae IS, Kwon DR (2015) Utility of sonoelastography in differentiating ruptured from unruptured epidermal cysts and implications for patient care. J Ultrasound Med 34(7): 1175-1181.

6. Itoh A, Ueno E, Tohno E, Kamma H, Takahashi H, et al. (2006) Breast disease: clinical applications of US elastography for diagnosis. Radiology 239(2): 341-350.

7. Nakashima K, Shiina T, Sakurai M, Enokido K, Endo T, et al. (2013) JSUM ultrasound elastography practice guidelines: breast. J Med Ultrason 40(4): 359-391. 
8. Rago T, Scutari M, Loiacono V, Santin F, Tonacchera M, et al. (2017) Low elasticity of thyroid nodules on ultrasound elastography is correlated with malignancy, degree of fibrosis, and high expression of galectin-3 and fibronectin-1. Thyroid 27(1): 103-110.

9. Patel K, Sellars ME, Clarke JL, Sidhu PS (2012) Features of testicular epidermoid cysts on contrast-enhanced sonography and real-time tissue elastography. J Ultrasound Med 31(1): 115-122.

10. Kim HK, Kim SM, Lee SH, Racadio JM, Shin MJ (2011) Subcutaneous epidermal inclusion cysts: ultrasound (US) and MR imaging findings. Skeletal Radiol 40(11): 1415-1419.

11. Jin W, Ryu KN, Kim GY, Kim HC, Lee JH, et al. (2008) Sonographic findings of ruptured epidermal inclusion cysts in superficial soft tissue: emphasis on shapes, pericystic changes, and pericystic vascularity. J Ultrasound Med 27(2): 171-176.

12. Wortsman X (2017) Sonography of dermatologic emergencies. J Ultrasound Med 36(9): 1905-1914.

13. Fujioka K (2018) Usefulness of comprehensive high-resolution ultrasound imaging in dermatologic field: epidermal cyst. Biomed J Sci \& Tec Res 12(1).

14. Lee YH, Song HT, Suh JS (2014) Use of strain ratio in evaluating superficial soft tissues tumors on ultrasonic elastography. J Med Ultrason 41(3): 319-323.

15. Park HJ, Lee SY, Lee SM, Kim WT, Lee S, et al. (2015) Strain elastography features of epidermoid tumours in superficial soft tissue: differences from other benign soft-tissue tumours and malignant tumours. $\mathrm{Br}$ Radiol 88 (1050): 20140797

16. Yeoh HJ, Kim TY, Ryu JA (2019) The feasibility of shear wave elastography for diagnosing superficial benign soft tissue masses. Ultrasonography 38(1): 37-43.

17. Fujioka K, Fujioka A, Eto H, Suzuki K, Sanuki E, et al. (2006) Nodular fascitis in the thigh followed up using ultrasonography. J Med Ultrason 33: 49-53.

18. Fujioka K, Fujioka A, Oishi M, Eto H, Tajima S, etal. (2017) Ultrasonography findings of intradermal nodular fasciitis; a rare case report and review of the literature. Clin Exp Dermatol 42(3): 335-336.

19. Fujioka K, Fujioka A, Tajima S, Oishi M, Hayashi K, et al. (2018) Characteristic power Doppler sonographic imaging of nodular fasciitis from dermatological perspective: another case and review of three cases. J Clin Case Rep 8(9): 1165.

20. Fujioka K, Fujioka A, Oishi M, Hayashi K, Nakayama T (2018) Highresolution ultrasound imaging for angioleiomyoma: a painful and vascularized superficial tumor. Biomed J Sci \& Tec Res 9(5).

\section{ISSN: 2574-1241}

\section{DOI: 10.26717/BJSTR.2019.16.002904}

Kazumi Fujioka. Biomed J Sci \& Tech Res

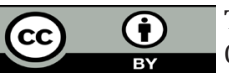

This work is licensed under Creative

Commons Attribution 4.0 License

Submission Link: https://biomedres.us/submit-manuscript.php
21. Fujioka K (2018) Presentations of clinical, ultrasonographic and pathological features of nodular fasciitis from an established cytogenetic viewpoint: review of the cases. J Carcinog Mutagen 9(4): 326.

22. Fujioka K (2019) Painful cutaneous and subcutaneous tumors accompanied with vascularized appearance using high-resolution ultrasound in dermatology: the acronym "ENGLAND" or "LEND AN EGG". Biomed J Sci \& Tec Res 12(4).

23. Egawa K, Egawa N, Honda Y (2005) Human papillomavirus-associated planar epidermoid cyst related to epidermoid metaplasia of the eccrine duct epithelium: a combined histological, immunohistochemical, DNA-DNA in situ hybridization and three-dimensional reconstruction analysis. Br J Dermatol 152(5): 961-967.

24. Lee S, Lee W, Chung S, Kim D, Sohn M, et al. (2003) Detection of human papillomavirus 60 in epidermal cysts of nonpalmoplantar location. Am J Dermatopathol 25(3): 243-247.

25. Sato Y, Nozaki T, Matsusako M, Eto H, Matsui M, et al. (2014) Human papillomavirus-associated plantar epidermoid cysts: MR and US imaging appearance. Skeletal Radiol 43(2): 257-261.

26. Huang CC, KO SF, Huang HY, Ng SH, Lee TY, et al. (2011) Epidermal cysts in the superficial soft tissue: sonographic features with an emphasis on the pseudotestis pattern. J Ultrasound Med 30(1): 11-17.

27. Lee HS, Joo KB, Song HT, Kim YS, Park DW, et al. (2001) Relationship between sonographic and pathologic findings in epidermal inclusion cysts. J Clin Ultrasound 29(7): 374-383.

28. Klauser AS, Miyamoto H, Bellmann Weiter R, Feuchtner GM, Wick MC, et al. (2014). Sonoelastography: musculoskeletal applications. Radiology 272(3): 622-633.

29. Bhatia KS, Rasalkar DD, Lee YP, Wong KT, King AD, et al. (2010) Realtime qualitative ultrasound elastography of miscellaneous non-nodal neck masses: applications and limitations. Ultrasound Med Biol 36(10) :1644-1652

30. Hong SH, Chung HW, Choi JY, Koh YH, Choi JA, et al. (2006) MRI findings of subcutaneous epidermal cysts: emphasis on the presence of rupture. AJR 186(4): 961-966.

31. Fisher AR, Mason PH, Wagenhais KS (1998) Ruptured plantar epidermal inclusion cyst. AJR 171(6): 1709-1710.

32. Vicente J, Vazquez Doval FJ (1998) Proliferation of the epidermal cyst wall. Int J Dermatol 37: 181-185.

33. Park JS, Ko DK (2013) A histopathologic study of epidermoid cysts in Korea: comparison between ruptured and unruptured epidermal cyst. Int J Clin Exp Pathol 6(2): 242-248.

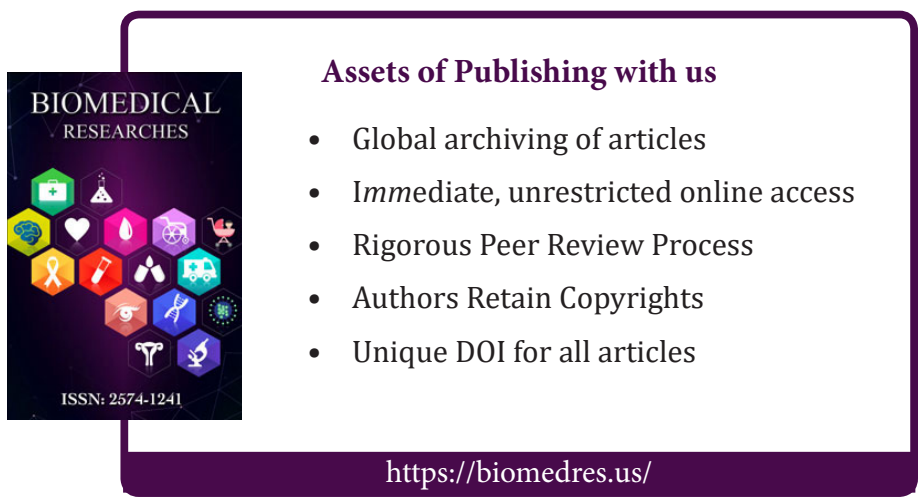

